\title{
Effects of Dietary N-Carbamylglutamate Supplementation on Milk Production Performance, Nutrient Digestibility and Blood Metabolomics of Lactating Holstein Cows Under Heat Stress
}

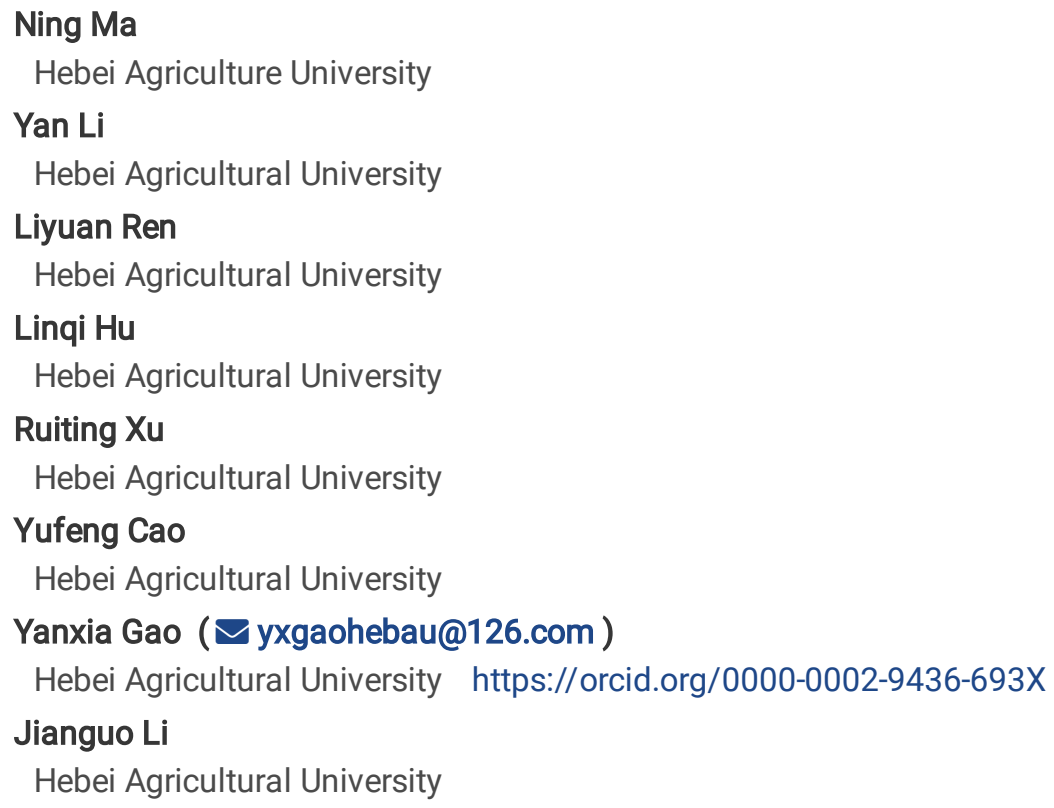

Research

Keywords: N-carbamylglutamate, Holstein cow, Milk, Metabonomic, Heat stress

Posted Date: May 29th, 2020

DOl: https://doi.org/10.21203/rs.3.rs-30617/v1

License: (c) (i) This work is licensed under a Creative Commons Attribution 4.0 International License. Read Full License

Version of Record: A version of this preprint was published at Animal Feed Science and Technology on March 1st, 2021. See the published version at https://doi.org/10.1016/j.anifeedsci.2020.114797. 


\section{Abstract}

Background: Heat stress (HS) becomes a serious threat to dairy cow industry in certain circumstances, N-carbamylglutamate (NCG) is a novel feed additive that may be used to alleviate HS. However, the effects and mechanisms of NCG on dairy cows under HS are still unknown. A total of 48 Holstein dairy cows with similar days in milk (154.37 $\pm 13.56 \mathrm{~d})$, parity (1-3), and body condition score (BCS) were randomly divided into 4 groups of 12 animals each. Under HS, the cows were fed a TMR diet supplemented with 0 (control), 15, 20, $25 \mathrm{~g}$ of NCG/d per cow for 60 days. Milk production performance was recorded and serum parameters were examined. Meanwhile, metabolomics study of plasma based on liquid chromatography-mass spectrometry (LC-MS) was also applied to investigate metabolites and pathways in response to NCG supplementation.

Results: Milk yield (MY) was increased but milk urine nitrogen (MUN) was reduced by the NCG treatment. Addition of $15 \mathrm{~g}$ of NCG/d increased the milk protein percentage (MPP) compared with the control. No effect of NCG was observed on dry matter intake (DMI), lactose percentage (LP), milk fat percentage (MFP) and somatic cell count (SCC). Serum glucose (GLU) levels in cows fed with 15, $20,25 \mathrm{~g}$ of NCG/d were increased by $14.35 \%, 19.34 \%$ and $18.63 \%$ in comparison with the control. Meanwhile, the concentrations of blood urea nitrogen (BUN) and blood ammonia (BA) were decreased with the addition of NCG. Results for antioxidant capacity and immune function showed beneficial effects of NCG, such as the increases in total antioxidant capacity (T-AOC), superoxide dismutase (SOD), glutathione peroxidase (GSH-Px), IgG, IgM and IgA. Clear separations of plasma metabolic profiles between control and NCG groups were observed in the score plots. Sixteen different metabolites involved in metabolisms of amino acids, ketone bodies, butanoate and energy, as well as gut microbiome-derived metabolism were regulated by NCG supplementation.

Conclusions: This study found that NCG treatment improved antioxidant capacity, immune function, production performance, and metabolic profile of dairy cows under HS and provided new evidence for the better utilization of NCG.

\section{Background}

Arginine participates in protein synthesis and metabolism to improve intestinal absorption function, hormones secretion and nitric oxide (NO) production, which ultimately benefits the growth, development and production performance of dairy cows [1, 2]. However, with the high arginase activity or rumen degradation, arginine is rapidly degraded with short biological half-time. Furthermore, the high dietary level of arginine may cause an absorption disorder of lysine due to the competition for the common transporters [3]. Moreover, arginine is uneconomically used in cows for its high cost. Due to these disadvantages, promoting the endogenous arginine synthesis will be an economic and alternative strategy to increase the supply of arginine for the animals.

The limiting factor in urea cycle of arginine synthesis is the availability of $\mathrm{N}$-acetylglutamate (NAG) which is unstable and easily hydrolyzed to acylamino acids [4]. N-carbamylglutamate (NCG) is an analogue of NAG, which can enter the mitochondria to activate carbamylphosphate synthase-1 (CPS-1) and therefore promote the synthesis of arginine. In addition, NCG is stable in the ruminal environment and dose not antagonize other amino acids such as lysine and histidine [5]. Thus, oral supplementation of NCG can increase the endogenous synthesis of arginine. At present, NCG as a promising feed additive, is widely used in farm animals production. Previous studies have found that dietary supplementation of NCG could promote the metabolism of urea cycle and the uptake and utilization of nitrogen in sheep [6]. Moreover, several results also suggested that NCG have positive effects on growth promotion, tissue development and reproductive performance in piglets, sheep, goats and broilers [7].

As global warming intensifying, heat stress (HS) in farm animals including the dairy cows becomes more problematic. When the temperature-humidity index ( $\mathrm{THI}$ ) value is greater than 72 , dairy cows will have a decline in dry mater intake (DMI) or even refuse to eat, which leads to a decreased milk yield (MY) [8]. Moreover, a series of metabolic disorders, such as mental malaise, anorexia, shortness of breath, acceleration of pulse times, and rise of body temperature, could also occur in the dairy cows under HS. All of these may lead detriments to the immunity and redox equilibrium [9]. Additionally, recent work has shown that milk composition and reproductive capacity of dairy cows are affected by HS besides the MY reduction [10].

Metabonomics studies the entire metabolic map and functional regulation in organism through the qualitative and quantitative analyses of the metabolites [11]. With high sensitivity and high resolving power, liquid chromatography-mass spectrometry (LC-MS)based metabolomics is now widely applied as a versatile tool to screen the changes in diverse biological systems [12, 13]. Through mining the metabolic pathways such as sugar generation, metabolism of amino acids and lipids, physiological status under diseases, stress and nutrients in dairy cows can be systematically evaluated by metabonomic method $[14,15]$. In dairy cows, main 
biofluid samples used in metabonomic studies are rumen fluid, blood and milk. Blood metabolites are easily affected and can be directly used to characterize metabolic pathways related with dietary treatment [16].

Although the physiological function of NCG has been demonstrated, its effects on dairy cows under HS remain unclear. This study attempted to increase the understanding of the physiological processes associated with NCG treatment and provide theoretical guidance for the application of NCG in dairy cows. The present study investigated the effects of dietary supplementation of NCG in dairy cows during lactation under HS. Milk yield and composition, blood biochemistry, HS indicators, antioxidant and immune indexes were evaluated in the experiment. With the application of LC-MS based metabonomics, the plasma metabolites associated with NCG treatment were identified.

\section{Methods}

\section{Animals, diets and experiment design}

NCG was produced by Beijing Animore Sci. \& Tech. Co., Ltd. (Beijing, China). Forty-eight Holstein dairy cows with similar days in milk (154.37 \pm 13.56 d), parity (1-3), and body condition score (BCS) were provided by Hong Da dairy farm (Baoding, Hebei). The cows were randomly separated into 4 groups ( $n=12$ per group) as: control (TMR diet), low dose NCG (LNCG, TMR with NCG $15 \mathrm{~g} / \mathrm{day}$ per cow), medium dose NCG (MNCG, TMR with NCG $20 \mathrm{~g} /$ day per cow) and high dose NCG (HNCG, TMR with NCG $25 \mathrm{~g} /$ day per cow). Experimental design was shown in Fig. 1. The detail composition and nutrient level of the basal diet were given in Table S1. The NCG treatment lasted for 60 days. THI was employed as an index of the HS status of the dairy cows. In this study, the THI of cowsheds ranged from 72 to 87.7 (Figure S1), indicating the dairy cows were clearly exposed to HS over the course of the experiment.

\section{Sample Collection And Measurements}

Samples of the TMR diets and orts were collected three times every week throughout the experiment period. Feed samples were dried $\left(65^{\circ} \mathrm{C}, 48 \mathrm{~h}\right)$ and ground for the analyses of $\mathrm{DMI}$, crude protein (CP), ether extract (EE), neutral detergent fiber (NDF), acid detergent fiber (ADF) [17], and contents of calcium (Ca) and phosphorus ( $P$ ). The measurement methods were accorded to the AOAC [18]. In addition, NDF and ADF were measured by the using of ANKOM-A2000i fiber analyzer (Ankom Technology, Macedon, NY).

Mile yield of each cow was recorded electronically three times every day. Milk samples were collected at the time points of $15,30,45$ and $60 \mathrm{~d}$ through milking at 0600,1400, and $2000 \mathrm{~h}$ in the experiment, and the samples were collected into the aliquot of $50 \mathrm{~mL}$ at the proportion of 4:3:3. Milk compositions including fat percentage (MFP), protein percentage (MPP), lactose percentage (LP), somatic cell count (SCC) and urea nitrogen (MUN) were analyzed at the DHI Central of Hebei Animal Husbandry Varieties Workstation (Shijiazhuang, China).

Blood samples $(10 \mathrm{~mL})$ were collected from the coccygeal vain into vacuum tubes with or without heparin before morning feeding at the middle (30 day) and end of the experiment ( 60 day), and then centrifuged $\left(2000 \mathrm{~g}, 4^{\circ} \mathrm{C}\right.$ and $\left.15 \mathrm{~min}\right)$ to obtain the serum and plasma, respectively. The plasma and serum samples were stored at $-80{ }^{\circ} \mathrm{C}$ until analysis. Serum was used to measure blood biochemistry indexes including glucose (GLU), urea nitrogen (BUN), total protein (TP), albumin (ALB), creatinine (CRE), aspartate aminotransferase (AST), alanine aminotransferase (ALT) and ammonia (BA) by the using of biochemistry analyzer Microlab-300. In addition, antioxidant and immune indexes and heat stress indicators were measured by the corresponding colorimetric methods or ELISA kits provided by Nanjing Jiancheng Biology Engineering Institute (Nanjing, China). Heat stress indicators were composed of triiodothyronine $\left(T_{3}\right)$, thyroxine $\left(T_{4}\right)$, growth hormone $(G H)$, prolactin (PRL), heat shock protein-70 (HSP-70), cortisol and ascorbic acid. Antioxidant indexes were consisted of total antioxidant capacity (T-AOC), glutathione peroxidase (GSH-Px), malondialdehyde (MDA) and superoxide dismutase (SOD). Immunoglobulins (Ig) including IgG, IgM and IgA were also examined.

\section{Sample Preparation For Metabonomics}

Plasma samples were thawed at room temperature prior to analysis. $200 \mu \mathrm{L}$ of plasma was placed into PE tubes, and then $400 \mu \mathrm{L}$ of methanol/water $(4: 1, \mathrm{v} / \mathrm{v})$ was added. Next, the tubes were vortex-mixed and incubated for $1 \mathrm{~h}$ at $-20^{\circ} \mathrm{C}$, and then centrifuged at $14,000 \mathrm{~g}$ for $20 \mathrm{~min}$ at $4{ }^{\circ} \mathrm{C}$. The supernatant was collected and dried. Lyophilized powder samples were reconstituted by dissolving 
in $100 \mu \mathrm{L}$ of $60 \%$ methanol. The samples were vortexed $(1 \mathrm{~min})$ and centrifuged $\left(14,000 \mathrm{~g}, 15 \mathrm{~min}, 4^{\circ} \mathrm{C}\right)$ to collect the supernatant for metabonomic analysis. Quality control (QC) samples prepared from the pooled supernatant were placed in the analysis sequence.

\section{Metabonomic Data Analysis}

Chromatographic separation of plasma was performed with Vanquish ultra high performance liquid chromatograph (UHPLC) system (Thermo, USA). An Accucore HILIC column $(2.1 \times 150 \mathrm{~mm}, 2.6 \mu \mathrm{m})$ was used in the study. The column was maintained at $40^{\circ} \mathrm{C}$, and the flow rate was $0.3 \mathrm{~mL} / \mathrm{min}$. The mobile phase for the positive ion mode was consisted of $A(0.1 \%$ formic acid, $95 \%$ acetonitrile, $10 \mathrm{mM}$ ammonium acetate) and $\mathrm{B}(0.1 \%$ formic acid, $50 \%$ acetonitrile, $10 \mathrm{mM}$ ammonium acetate); the negative ion mode was consisted of A ( $95 \%$ acetonitrile, $10 \mathrm{mM}$ ammonium acetate) and B (50\% acetonitrile, $10 \mathrm{mM}$ ammonium acetate). The process of linear gradient elution was shown as follows: 0-1 min, 98\% $A ; 1-17 \mathrm{~min}, 98-50 \% A ; 17-17.5 \mathrm{~min}, 50 \% \mathrm{~A} ; 17.5-18 \mathrm{~min}, 50-98 \% A$; 18-20 min, $98 \%$ A.

Mass spectrometer used in this study was Q Exactive ${ }^{\mathrm{TM}} \mathrm{HF}-\mathrm{X}$ (Thermo, USA) equipped with an electrospray ionization source (ESI) operating in positive (ESI+) and negative ion modes (ESI-). The MS main properties were set as follows: spray voltage, $3.2 \mathrm{KV}$; sheath gas flow rate, 35 arb; aux gas flow rate, 10 arb; scan range, $\mathrm{m} / \mathrm{z}$ of $100-1500$; capillary temperature, $320^{\circ} \mathrm{C}$. MS/MS data was acquired in data dependent acquisition (DDA) mode.

\section{Metabonomic data processing and metabolites identification}

The MS data was processed by Compound Discovery 2.0 (Thermo, USA) for nonlinear alignment, automatic integration and extraction of the peak intensities. Then, the obtained dataset was imported into SIMCA-P (Umetrics AB, Sweden) for multivariate statistical analysis. Principal component analysis (PCA) and partial least squares discriminant analysis (PLS-DA) were performed. Cumulative $\mathrm{R}^{2}$ and $\mathrm{Q}^{2}$ of the PCA and OPLS-DA models were used to evaluate the fitness and predictive capability of the model. Variable importance in projection (VIP) score in the OPLS-DA model was constructed, and the $P$-value of the candidate metabolite was analyzed by Student's t-test. Metabolite with VIP $>1$ and $P<0.05$ was considered to be statistically significant.

According to the exact molecular weight and MS/MS fragmentation pattern data, metabolite identification was carried out through the database search including HMDB (http://www.hmdb.ca/), METILN (https://metlin.scripps.edu), mzCloud

(https://www.mzcloud.org/), and Chemspider (http://www.chemspider.com/). KEGG (https://www.kegg.jp/) and HMDB search was conducted to identify the metabolic pathways and biochemical reactions of the metabolites. Pathway analysis was performed by MetaboAnalyst 4.0 (http://www.metaboanalyst.ca/) for further biological interpretation.

\section{Statistical analysis}

All the data were analyzed using the MIXED procedure in the SAS software version 9.4 (SAS Institute Inc., Cary, NC). In MIXED model, NCG supplementations were fixed effects and cows were random effects. The PDIFF option adjusted by the Tukey method was included in the LSMEANS statement to account for multiple comparisons among treatments. The linear and quadratic responses to increasing NCG supplementation doses were determined by using specific preplanned contrasts. Data were presented as mean and mean standard error (SEM). Treatment effects were declared significant at $P \leq 0.05$, and trends were discussed at $0.05<P \leq 0.10$.

\section{Results}

\section{Milk production performance and nutrients apparent digestibility}

NCG treatment significantly increased MY and reduced MUN $(P<0.05)$ (Table 1). Compared with the control, MPP in LNCG increased by $7.36 \%$. Meanwhile, low, medium, and high doses of NCG reduced MUN by $17.73 \% \otimes 13.46 \% \bigotimes 16.31 \%$, respectively, as compared with the control. No significant difference was observed among DMI, LP, SCC, and MFP among NCG-treated groups and control. Quadratic response to the increasing NCG was observed for MY $(P=0.04)$, MPP $(P=0.01)$, and MUN $(P=0.09)$. Results of the MY suggested that NCG at the dosage of $20 \mathrm{~g}$ /day per cow was optimal. In addition, NCG had no effects on the nutrients apparent digestibility $(P>$ 0.05) (Table S2). 
Table 1

Effects of different NCG addition on DMI and performance of lactating Holstein cows.

\begin{tabular}{|c|c|c|c|c|c|c|c|c|}
\hline \multirow[t]{2}{*}{ Items } & \multicolumn{4}{|l|}{ Groups } & \multirow[t]{2}{*}{ SEM } & \multicolumn{3}{|c|}{ Probability } \\
\hline & Control & LNCG & MNCG & HNCG & & P-NCG & Linear & Quadratic \\
\hline DMI, kg/d & 22.67 & 23.42 & 23.21 & 22.70 & 1.16 & 0.96 & 0.89 & 0.59 \\
\hline$M Y, k g / d$ & $29.90^{\mathrm{b}}$ & $31.32^{\mathrm{a}}$ & $31.48^{\mathrm{a}}$ & $31.20^{\mathrm{a}}$ & 0.278 & $\varangle 0.01$ & $\triangle 0.01$ & 0.04 \\
\hline MFP, \% & 3.64 & 3.73 & 3.70 & 3.67 & 0.081 & 0.90 & 0.69 & 0.52 \\
\hline MPP, \% & $3.27^{b}$ & $3.49^{a}$ & $3.36^{\mathrm{ab}}$ & $3.35^{b}$ & 0.046 & 0.03 & 0.18 & 0.01 \\
\hline LP, \% & $5.00^{\mathrm{ab}}$ & $5.02^{a}$ & $4.94^{b}$ & $4.95^{\mathrm{ab}}$ & 0.026 & 0.10 & 0.10 & 0.26 \\
\hline $\mathrm{ScC}, 10^{4} / \mathrm{ml}$ & $4.65^{\mathrm{a}}$ & $3.89^{a b}$ & $3.33^{b}$ & $3.65^{\mathrm{ab}}$ & 0.381 & 0.09 & 0.02 & 0.54 \\
\hline MUN, mg/dL & $13.37^{\mathrm{a}}$ & $11.00^{\mathrm{b}}$ & $11.57^{\mathrm{b}}$ & $11.19^{\mathrm{b}}$ & 0.431 & $\varangle 0.01$ & $\otimes 0.01$ & 0.09 \\
\hline
\end{tabular}

\section{Blood Biochemistry}

The levels of GLU in LNCG, MNCG and HNCG were significantly higher than those in the control with increasing by $14.35 \%, 19.34 \%$ and $18.63 \%(P<0.05)$ (Table S3). The BUN and BA were markedly reduced in NCG treated groups $(P<0.01)$. Meanwhile, the levels of GLU and BA were linearly elevated and decreased, respectively $(P<0.01)$, in response to increase of NCG dosage. There were no significant differences in the TP, ALB, CRE, ALT, and AST among groups.

\section{Antioxidant And Immune Indexes}

As shown in Table 2, in comparison with the control group, the T-AOC, SOD, and GSH-Px levels were significantly increased $(P<0.05)$, and the MDA was markedly decreased in three NCG treatment groups $(P<0.05)$. T-AOC, SOD, and GSH-Px increased linearly $(P<0.01)$ and MDA decreased linearly $(P<0.01)$ with increasing NCG dosages. The levels of T-AOC, SOD, and GSH-Px in HNCG group were significant higher and MDA was lower than those in LNCG or MNCG groups $(P<0.05)$, which showed a dose-effect relationship between antioxidant indexes and NCG. It was found that the values of IgA and IgG in all NCG groups were higher than those in the control $(P<0.05)$. The levels of IgM were not different among control, LNCG and HNCG, but it was markedly higher in MNCG $(P<$ 0.05). IgA and IgM showed a quadratic response $(P<0.01)$ and IgG was linearly increased $(P<0.01)$ with increasing dosages of NCG. Moreover, the high values of IgG, IgA, and IgM in MNCG group suggest an optimal NCG dosage for immunity enhancement. 
Table 2

Effect of NCG on antioxidant and immune indexes.

\begin{tabular}{|c|c|c|c|c|c|c|c|c|}
\hline \multirow[t]{2}{*}{ Items } & \multicolumn{4}{|l|}{ Groups } & \multirow[t]{2}{*}{ SEM } & \multicolumn{3}{|c|}{ Probability } \\
\hline & Control & LNCG & MNCG & HNCG & & $P$-NCG & Linear & Quadratic \\
\hline T-AOC, U/mL & $34.14^{c}$ & $43.74^{\mathrm{b}}$ & $45.49^{b}$ & $48.41^{\mathrm{a}}$ & 0.960 & $\otimes 0.01$ & $\otimes 0.01$ & 0.47 \\
\hline $\mathrm{SOD}, \mathrm{ng} / \mathrm{mL}$ & $6.66^{c}$ & $8.77^{b}$ & $9.64^{\mathrm{a}}$ & $10.18^{a}$ & 0.242 & $\varangle 0.01$ & $\bowtie 0.01$ & 0.87 \\
\hline GSH-Px, ng/mL & $3,277^{c}$ & $3,621^{b}$ & $4,343^{a}$ & $4,501^{a}$ & 83.87 & $\varangle 0.01$ & $\llbracket 0.01$ & $凶 0.01$ \\
\hline $\mathrm{MDA}, \mathrm{mmol} / \mathrm{mL}$ & $8.25^{\mathrm{a}}$ & $7.26^{b}$ & $6.75^{b}$ & $4.55^{c}$ & 0.184 & $\varangle 0.01$ & $\bowtie 0.01$ & $\otimes 0.01$ \\
\hline $\lg A, \mathrm{mg} / \mathrm{mL}$ & $13.33^{d}$ & $15.53^{c}$ & $25.06^{a}$ & $23.15^{b}$ & 0.477 & $\otimes 0.01$ & $\varangle 0.01$ & $\nabla 0.01$ \\
\hline $\mathrm{IgM}, \mathrm{mg} / \mathrm{mL}$ & $11.80^{\mathrm{b}}$ & $12.23^{b}$ & $15.34^{\mathrm{a}}$ & $11.89^{b}$ & 0.360 & $\otimes 0.01$ & 0.01 & $凶 0.01$ \\
\hline $\mathrm{IgG}, \mathrm{mg} / \mathrm{mL}$ & $23.30^{d}$ & $26.49^{c}$ & $30.28^{a}$ & $28.00^{b c}$ & 0.658 & $\otimes 0.01$ & $₫ 0.01$ & 0.12 \\
\hline
\end{tabular}

\section{Hs Indicators}

As shown in Table 3, the values of $\mathrm{T}_{3}, \mathrm{GH}, \mathrm{PRL}$, and ascorbic acid were significantly higher but cortisol was markedly lower in all three NCG groups than that in the control $(P<0.05)$. There were no differences in HSP-70 and $\mathrm{T}_{4}$ between control and the NCG treatment groups. Linear responses to increasing NCG dosages were obtained for $\mathrm{T}_{3}, \mathrm{PRL}, \mathrm{HSP}-70$, cortisol, and ascorbic acid $(P<$ 0.01). Meanwhile, NCG had quadratic significant effect on GH $(P<0.01)$. Notably, the levels of HSP-70, ascorbic acid, and $T_{3}$ in MNCG and HNCG were higher and the cortisol was lower than those in the LNCG $(P<0.05)$.

Table 3

Effect of NCG on heat stress indicators.

\begin{tabular}{|c|c|c|c|c|c|c|c|c|}
\hline \multirow[t]{2}{*}{ Items } & \multicolumn{4}{|l|}{ Groups } & \multirow[t]{2}{*}{ SEM } & \multicolumn{3}{|c|}{ Probability } \\
\hline & Control & LNCG & MNCG & HNCG & & PNCG & Linear & Quadratic \\
\hline Cortisol, ng/mL & $119.18^{a}$ & $96.00^{\mathrm{b}}$ & $68.97^{c}$ & $74.73^{\mathrm{c}}$ & 2.25 & $\varangle 0.01$ & $\nabla 0.01$ & 0.63 \\
\hline $\mathrm{T}_{3}, \mathrm{nmol} / \mathrm{L}$ & $2.77^{d}$ & $4.30^{c}$ & $4.90^{\mathrm{b}}$ & $5.63^{\mathrm{a}}$ & 0.124 & $\varangle 0.01$ & $\otimes 0.01$ & 0.19 \\
\hline $\mathrm{T}_{4}, \mathrm{nmol} / \mathrm{L}$ & 160.36 & 157.87 & 151.99 & 156.32 & 5.19 & 0.71 & 0.40 & 0.81 \\
\hline $\mathrm{GH}, \mathrm{ng} / \mathrm{mL}$ & $7.61^{\mathrm{c}}$ & $10.52^{b}$ & $11.84^{\mathrm{a}}$ & $10.51^{\mathrm{b}}$ & 0.288 & $\nabla 0.01$ & $凶 0.01$ & $\varangle 0.01$ \\
\hline $\mathrm{PRL}, \mathrm{mlU} / \mathrm{L}$ & $342.70^{c}$ & $453.70^{b}$ & $461.47^{b}$ & $487.62^{a}$ & 8.55 & $\varangle 0.01$ & $\nabla 0.01$ & 0.05 \\
\hline HSP-70, pg/mL & $252.19^{a b}$ & $247.58^{b}$ & $280.81^{a}$ & $268.63^{a}$ & 6.25 & $\varangle 0.01$ & 0.01 & 0.32 \\
\hline Ascorbic acid, $\mathrm{ng} / \mathrm{mL}$ & $14.58^{\mathrm{c}}$ & $16.33^{\mathrm{b}}$ & $19.23^{\mathrm{a}}$ & $19.79^{a}$ & 0.385 & $₫ 0.01$ & $\varangle 0.01$ & 0.03 \\
\hline
\end{tabular}

\section{Metabolites Identification Associated With Ncg Treatment}

In order to identify the metabolites related with the NCG treatment, the PLS-DA models were established. According to the PLS-DA score plots in ESI + and ESI- modes, a clear separation between control and NCG-treated groups was observed (Figure S3). Either in 
PCA or PLS-DA score plots, it was found that the separation trend between control and NCG-treated groups was good, which indicated that the NCG could cause the level changes of some potential metabolites. Based on the VIP $>1$ and $P<0.05$, the different metabolites were selected. Sixteen different plasma metabolites associated with NCG were listed in Table 4. It was found that the concentrations of 8 metabolites significantly increased and 8 metabolites remarkably decreased in NCG-treated groups compared with the control (Table 4). Based on the metabolite abundance, the hierarchical clustering analysis was carried, and the identified metabolites were visualized in a clustering heatmap (Fig. 2A). In the heat map, the dendrogram showed that the samples in the control group were clustered, and the samples in NCG-treated groups were separated from the control. Meanwhile, the metabolites with similar abundance patterns were also closely positioned.

Table 4

Significantly modulated metabolites in cows related with NCG based on PLS-DA.

\begin{tabular}{|c|c|c|c|c|c|c|c|c|}
\hline \multirow[t]{2}{*}{ No. } & \multirow[t]{2}{*}{ Formula } & \multirow[t]{2}{*}{ MW } & \multirow[t]{2}{*}{ RT } & \multirow[t]{2}{*}{ Metabolite } & \multicolumn{3}{|c|}{ Fold Change } & \multirow[t]{2}{*}{ KEGG Pathway } \\
\hline & & & & & LNCG/C & MNCG/C & HNCG/C & \\
\hline 1 & $\mathrm{C}_{6} \mathrm{H}_{14} \mathrm{~N}_{2} \mathrm{O}_{2}$ & 146.10529 & 0.91 & Lysine & 0.90 & $0.51^{*}$ & 0.56 & Lysine metabolism \\
\hline 2 & $\mathrm{C}_{5} \mathrm{H}_{10} \mathrm{O}_{3}$ & 118.0628 & 1.713 & 3-Hydroxyvaleric acid & 0.82 & $0.65^{*}$ & 0.84 & - \\
\hline 3 & $\mathrm{C}_{22} \mathrm{H}_{44} \mathrm{NO}_{7} \mathrm{P}$ & 465.2842 & 13.555 & LysoPC (14:1) & 1.36 & $1.80^{\star \star}$ & 1.00 & $\begin{array}{l}\text { Glycerophospholipid } \\
\text { metabolism }\end{array}$ \\
\hline 4 & $\mathrm{C}_{20} \mathrm{H}_{34} \mathrm{O}_{2}$ & 306.255 & 14.518 & Linolenic acid & 0.88 & 1.09 & $0.61^{*}$ & $\begin{array}{l}\text { Linoleic acid } \\
\text { metabolism }\end{array}$ \\
\hline 5 & $\mathrm{C}_{12} \mathrm{H}_{22} \mathrm{O}_{4}$ & 230.15093 & 11.976 & Dodecanedioic acid & $1.29^{\star \star}$ & $1.62^{*}$ & $1.49^{\star \star}$ & - \\
\hline 6 & $\mathrm{C}_{26} \mathrm{H}_{43} \mathrm{NO}_{6}$ & 465.3075 & 12.868 & Glycocholic acid & $2.08^{*}$ & $2.03^{\star \star}$ & $2.03^{*}$ & $\begin{array}{l}\text { Primary bile acid } \\
\text { biosynthesis }\end{array}$ \\
\hline 7 & $\mathrm{C}_{4} \mathrm{H}_{5} \mathrm{~N}_{3} \mathrm{O}$ & 111.04307 & 1.087 & Cytosine & 1.30 & 0.89 & $1.56^{\star *}$ & $\begin{array}{l}\text { Pyrimidine } \\
\text { metabolism }\end{array}$ \\
\hline 8 & $\mathrm{C}_{9} \mathrm{H}_{8} \mathrm{O}_{3}$ & 164.04707 & 1.391 & $\begin{array}{l}\text { 2-Hydroxycinnamic } \\
\text { acid }\end{array}$ & 0.74 & $0.51^{\star \star}$ & 0.86 & $\begin{array}{l}\text { Phenylalanine } \\
\text { metabolism }\end{array}$ \\
\hline 9 & $\mathrm{C}_{9} \mathrm{H}_{8} \mathrm{O}_{2}$ & 148.0528 & 0.38 & Cinnamic acid & 1.29 & $1.90^{*}$ & 2.88 & - \\
\hline 10 & $\mathrm{C}_{16} \mathrm{H}_{30} \mathrm{O}_{2}$ & 254.2245 & 0.211 & Palmitelaidic acid & 0.60 & $0.29^{*}$ & 0.48 & - \\
\hline 11 & $\mathrm{C}_{6} \mathrm{H}_{11} \mathrm{NO}_{2}$ & 129.0792 & 1.59 & Pipecolic acid & 0.85 & $0.65^{\star \star}$ & 1.03 & Lysine degradation \\
\hline 12 & $\mathrm{C}_{26} \mathrm{H}_{43} \mathrm{NO}_{5}$ & 449.3148 & 12.866 & $\begin{array}{l}\text { Glycoursodeoxycholic } \\
\text { acid }\end{array}$ & $1.81^{*}$ & $1.81^{* *}$ & 1.66 & - \\
\hline 13 & $\mathrm{C}_{4} \mathrm{H}_{7} \mathrm{~N}_{3} \mathrm{O}$ & 113.0592 & 0.088 & Creatinine & $1.49^{*}$ & $1.56^{* *}$ & 2.49 & $\begin{array}{l}\text { Arginine and proline } \\
\text { metabolism }\end{array}$ \\
\hline 14 & $\mathrm{C}_{24} \mathrm{H}_{40} \mathrm{O}_{6}$ & 424.2832 & 11.813 & Hydroxycholic acid & $1.99^{*}$ & 2.49 & $1.78^{*}$ & - \\
\hline 15 & $\mathrm{C}_{11} \mathrm{H}_{12} \mathrm{~N}_{2} \mathrm{O}_{2}$ & 204.0903 & 5.36 & Tryptophan & $0.59^{*}$ & 0.84 & 0.84 & $\begin{array}{l}\text { Amino acid } \\
\text { metabolism }\end{array}$ \\
\hline 16 & $\mathrm{C}_{4} \mathrm{H}_{8} \mathrm{O}_{3}$ & 104.0476 & 1.097 & 3-Hydroxybutyric acid & 0.87 & $0.66^{*}$ & 0.87 & $\begin{array}{l}\text { Butanoate } \\
\text { metabolism }\end{array}$ \\
\hline
\end{tabular}

RT: retention time; MW: molecular weight; -: not available in the KEGG pathway; Compared with the control, * $P<0.05$, ${ }^{\star \star} P<0.01$.

\section{Pathway Analysis}

The metabolites were checked against the KEGG database to find out the corresponding metabolic pathways and the biological pathways related with the metabolites were listed in Table 4. The pathway analysis was also performed by MetaboAnalyst to identify and visualize the metabolic pathway associated with NCG treatment. The summary of the pathway analysis results was shown in 
Table S4. Figure 2B showed that the pathways in response to NCG treatment were lysine degradation, synthesis and degradation of ketone bodies, aminoacyl-tRNA biosynthesis, metabolisms of linolenic acid, butanoate, glycerophospholipid, and tryptophan.

\section{Discussion}

HS in dairy cows causes reductions in milk yield and quality and reproductive performance, which lead to a tremendous economic loss to dairy cow producers. Moreover, the metabolomic disturbances induced by HS also make the dairy cows more susceptible to diseases [19]. Severe energy reduction caused by reduced DMI is responsible for the above changes of the dairy cows under HS [20]. However, some studies suggested that HS-induced decrease in DMI accounted for only approximately $35-50 \%$ of the MY reduction [21]. In present study, the DMI in NCG-treated groups were tended higher than in the control, but not significant. The results of markedly increased MY in NCG groups under HS are consistent with previous findings that lactation performance of dairy cows could be improved by the NCG supplementation at $20 \mathrm{~g} /$ day per cow [22]. It suggests that the increased MY in NCG groups may be not closely related with DMI, but through the improvement of factors in blood biochemistry, oxidative stress, and serum metabonomic profile.

Arginine has been found to increase the milk protein yield and protein content in dairy cows. Supplementation of NCG can increase the concentration of arginine in blood and promote the synthesis of milk protein. In this study, the increase in MPP in NCG treatment groups was agreed with previous reports [23]. It was found that the MPP values in LNCG group were significantly increased compared with the control. This suggests that $15 \mathrm{~g} \mathrm{NCG/day} \mathrm{to} \mathrm{the} \mathrm{cows} \mathrm{is} \mathrm{sufficient} \mathrm{to} \mathrm{enhance} \mathrm{the} \mathrm{endogenous} \mathrm{synthesis} \mathrm{of}$ arginine. The decreases in levels of MUN, BUN and BA in blood in NCG groups indicate that NCG might improve the milk protein through the utilization of urea synthesis. Previous studies reported that HS affected the energy metabolism through the reduction of GLU concentration [24]. The increase of GLU in NCG groups in this study may be beneficial for the enhancement of energy metabolism and milk synthesis. Gu et al found that NCG addition could increase the milk fat content in mid-lactating dairy cows [23]. However, the MFP of the dairy cows under HS showed no changes between control and NCG groups in this study. The reason for this inconsistence might be attributed to the different physiological status and lactation stages.

HS can cause damage to immunity and induce oxidative stress in dairy cows [25]. Compromised immune function was also observed that the serum IgG concentration in calves under HS was significantly reduced [26]. Oxidative stress not only induces DNA damage and mitochondrial disorder, but also affects the protein synthesis and gene expression. Antioxidants including T-AOC, GSHPx and SOD have been known to neutralize the detrimental effects of oxidative stress [27]. According to the results, the protective effects of NCG were detected where NCG supplementation increased the concentrations of T-AOC, GSH-Px and SOD and reduced the MDA in plasma of the cows under HS. These findings are consistent with those in the previous studies [28, 29]. Levels of immunoglobulins in serum are important indicators of immune response. Zhang et al reported that NCG could improve immune function in the intrauterine growth retarded lambs by the elevated plasma IgA concentration [30]. Consistent results were observed in present study that the levels of $\lg A$, IgM, and IgG were elevated by NCG treatment indicating the improvement of immunity of dairy cows. The above findings demonstrate that NCG supplementation attenuated HS in dairy cows by increasing the production of antioxidants and the enhancement of immune response.

Various hormones, such as $T_{3}, T_{4}$ and cortisol released from the endocrine system, are important to maintain the balance of body heat production and lose. Reduction of $T_{3}$, increase of cortisol and the overexpression of HSP-70 are used as indicators for the animal exposure to HS [31]. Compared with the control, NCG significantly reduced the levels of cortisol and increased $\mathrm{T}_{3}$ in this study. This indicates the counteracting of negative effects from HS. GH and PRL are involved in the energy metabolism in response to HS. Therefore, these hormones may be used to enhance the milk production. HS suppresses the production of GH and PRL and caused the alteration of energy metabolism [32]. Higher plasma concentrations of GH and PRL in NCG-treated cows than those in the control may explain the enhanced energy metabolism for milk synthesis and improved the productive performance of dairy cows under HS.

Endogenous metabolite alterations are associated with milk production reduction induced by HS [33]. In the present study, LC-MS based metabolomics was performed, and the metabolic profiling of plasma was analyzed to screen the metabolites related with NCG treatment. Sixteen different metabolites were identified and the pathway analysis indicated that these metabolites were potentially involved in metabolisms of amino acids, ketone bodies, butanoate, energy as well as gut microbiome-derived metabolism (Fig. 8). These findings help us to understand the underlying physiological mechanisms of NCG on dairy cows under HS. 
NCG affected amino acid metabolism of dairy cows under HS. Tian et al found that HS could increase concentrations of amino acids in plasma such as lysine, arginine and isoleucine through the regulation of nitrogen metabolism or energy metabolism [34]. In present study, the changes of amino acids induced by HS were reversed by the NCG treatment where the levels of lysine, tryptophan and pipecolic acid were reduced. Consistent with our partial results, a previous finding revealed that NCG significantly reduced plasma lysine concentration in rats suffered with oxidative stress [35]. In present study, reduced levels of lysine, tryptophan and pipecolic acid in plasma were consistent with the function of NCG in increasing the MPP in cows. Milk protein synthesis can decrease the level of amino acids in plasma due to that the amino acids are converted into proteins. It was found that NCG could increase protein synthesis and nitrogen utilization in animals [22]. Therefore, NCG might increase the MPP through sufficient using of amino acids in dairy cows under HS.

Creatinine as a degradation product of creatine phosphate plays an important role in maintaining energy homeostasis. Increased concentration of creatinine in NCG treatment groups suggested that creatine phosphate mobilization was increased to satisfy the energy requirement in response to HS [36]. 3-hydroxybutyric acid is a ketone body, and its level can be increased with the promotion of fatty acid $\beta$-oxidation. The decreased level of 3-hydroxybutyric acid in the NCG treatment groups might be caused through the improvement of energy metabolism. Under the HS, decreased level of GLU implied that the energy production from glucose aerobic oxidation was inhibited, and the $\beta$-oxidation of fatty acids might be promoted to increase the energy production [37]. NCG treatment showed favorable inhibition of 3-hydroxybutyric acid indicating the depression of fatty acid $\beta$-oxidation. This might be related to the improvement of energy metabolism. Dodecanedioic acid is a dicarboxylic acid. It is rapidly oxidized in the peroxisomes and then degraded in the mitochondria by $\beta$-oxidation [38]. Marked increase of dodecanedioic acid in NCG groups also indicated the reduction of fatty acid $\beta$-oxidation. Notably, results of 3-hydroxybutyric acid agreed with the increase of dodecanedioic acid. According to these results, it was speculated that NCG could maintain energy homeostasis of the dairy cows in response to HS by meliorating the changes of dodecanedioic acid, creatinine and 3-hydroxybutyric acid.

As physiological detergent, bile acids are essential for the excretion, absorption, and transport of fats in the liver and intestine. This study found that NCG increased the concentrations of glycocholic, glycoursodeoxycholic and hydroxycholic acids. The increase of these bile acids in the NCG group could facilitate the digestion and intestinal absorption of hydrophobic nutrients, which might make positive effects for dairy cows against HS. Different species of bacteria such as Bacteroides, Clostridium and Lactobacillus are involved in bile acid metabolism, and thus the gut microflora can modify bile acid composition [39]. Alterations of these bile acids might also be associated with the interventional effects of NCG on gut microbiome-derived metabolism, which should be investigated in future studies. It has been found that cinnamic acid and its derivatives have anti-oxidative activities [40]. NCG improved cinnamic acid level in plasma compared with the control. This agreed with the improvement of antioxidant indexes and was beneficial for improving the ability of antioxidants from NCG to decrease the oxidative stress injury. However, the level of 2hydroxycinnamic acid was reduced in NCG groups. The possible reason for this result was that chemical structure instability of 2hydroxycinnamic acid which was an extremely weak basic compound, and NCG might aggravate its consumption to produce antioxidative effects.

\section{Conclusions}

The present study suggested that supplementation with NCG in dairy cows could effectively alleviate HS, enhance antioxidant capacity, and immune function. These were evidenced by the improvement of HS indicators, antioxidant and immune indexes. These changes were contributed to improving the production performance of the dairy cows under HS. In addition, sixteen metabolites were identified by the LC-MS metabonomic analysis indicating that NCG altered some metabolic processes, such as metabolisms of energy, amino acids and gut microbiome-derived metabolism. Metabolic pathway analysis provided new insights into the biological function of NCG in dairy cows under HS. This study provided new evidence for the better utilization of NCG in dairy cow industry.

\section{Abbreviations}

HS: heat stress; NCG: N-carbamylglutamate; BCS: body condition score; LC-MS: liquid chromatography-mass spectrometry; MY: milk yield; MUN: milk urine nitrogen; MPP: milk protein percentage; DMI: dry matter intake; LP: lactose percentage; MFP: milk fat percentage; SCC: somatic cell count; GLU: glucose; MUN: milk urea nitrogen; BUN: blood urea nitrogen; T-AOC: total antioxidant capacity; SOD: superoxide dismutase; GSH-Px: glutathione peroxidase; NO: nitric oxide; THI: temperature-humidity index; CP: crude protein; EE: ether extract; NDF: neutral detergent fiber; ADF: acid detergent fiber; BUN: blood urea nitrogen; TP: total protein; ALB: 
albumin; CRE: creatinine; AST: aspartate aminotransferase; ALT: alanine aminotransferase; BA: blood ammonia; $\mathrm{T}_{3}$ : triiodothyronine; $\mathrm{T}_{4}$ : thyroxine; GH: growth hormone; PRL: prolactin; HSP-70: heat shock protein-70; MDA: malondialdehyde; ESI: electrospray ionization source; PCA: principal component analysis; PLS-DA: partial least squares discriminant analysis; VIP: variable importance in projection.

\section{Declarations}

\section{Acknowledgement}

The authors thank the entire stuff of Hongda Dairy Farm for their assistance in animal care and sample collection.

\section{Author contributions}

YXG and JGL designed the experiments. YL, LYR, and RTX conducted the experiment. YFC and NM prepared materials. LQH, RTX, NM and $Y L$ prepared the manuscript. YXG and JGL reviewed and improved the manuscript. All authors read and approved the final manuscript.

\section{Funding}

This work was supported by the earmarked fund by China Agriculture Research System (CARS-36), Hebei Dairy Cattle Innovation Team of Modern Agro-industry Technology Research System (HBCT2018120203).

\section{Availability of data and materials}

The datasets used and/or analyzed during the current study available from the corresponding author on reasonable request.

\section{Ethics approval and consent to participate}

The protocol for animal study was approved by Institutional Animal Care and Use Committee of Hebei Agricultural University and adhered to the guidelines of the Care and Use of Laboratory Animals of China.

\section{Consent to publish}

Not applicable

\section{Competing interests}

The authors declare that they have no competing interests

\section{References}

1. Gu FF, Liang SL, Wei ZH, Wang CP, Liu HY, Liu JX, et al. Short communication: Effects of dietary addition of Ncarbamoylglutamate on milk composition in mid-lactating dairy cows. J Dairy Sci. 2018;101(12):10985-90.

2. Zhao FF, Wu TY, Wang HR, Ding LY, Ahmed G, Li HW, et al. Jugular arginine infusion relieves lipopolysaccharide-triggered inflammatory stress and improves immunity status of lactating dairy cows. J Dairy Sci. 2018;101(7):5961-70.

3. Palencia JYP, Saraiva A, Abreu MLT, Zangeronimo MG, Schinckel AP, Pospissil Garbossa CA. Effectiveness of citrulline and Ncarbamoyl glutamate as arginine precursors on reproductive performance in mammals: A systematic review. Plos One. 2018;13(12):e209569.

4. Shi D, Allewell NM, Tuchman M. The N-Acetylglutamate Synthase Family: Structures, Function and Mechanisms. Int J Mol Sci. 2015;16(6):13004-22.

5. Ma Y, Zeng Z, Kong L, Chen Y, He P. Determination of N-Carbamylglutamate in Feeds and Animal Products by High Performance Liquid Chromatography Tandem Mass Spectrometry. Molecules. 2019;24(17):3172.

6. Oba M, Baldwin RLT, Owens SL, Bequette BJ. Metabolic fates of ammonia-N in ruminal epithelial and duodenal mucosal cells isolated from growing sheep. J Dairy Sci. 2005;88(11):3963-70. 
7. Zeng X, Huang Z, Zhang F, Mao X, Zhang S, Qiao S. Oral administration of N-carbamylglutamate might improve growth performance and intestinal function of suckling piglets. Livest Sci. 2015;181:242-8.

8. Jeelani R, Konwar D, Khan A, Kumar D, Chakraborty D, Brahma B. Reassessment of temperature-humidity index for measuring heat stress in crossbred dairy cattle of a sub-tropical region. J Therm Biol. 2019;82:99-106.

9. Zhang FJ, Weng XG, Wang JF, Zhou D, Zhang W, Zhai CC, et al. Effects of temperature-humidity index and chromium supplementation on antioxidant capacity, heat shock protein 72, and cytokine responses of lactating cows. J Anim Sci. 2014;92(7):3026-34.

10. Sun LL, Gao ST, Wang K, Xu JC, Sanz-Fernandez MV, Baumgard LH, et al. Effects of source on bioavailability of selenium, antioxidant status, and performance in lactating dairy cows during oxidative stress-inducing conditions. J Dairy Sci. 2019;102(1):311-9.

11. Zhang A, Sun H, Yan G, Wang P, Wang X. Mass spectrometry-based metabolomics: applications to biomarker and metabolic pathway research. Biomed Chromatogr. 2016;30(1):7-12.

12. Rezzi S, Collino S, Goulet L, Martin F. Metabonomic approaches to nutrient metabolism and future molecular nutrition. TrAC Trend Anal Chem. 2013;52:112-9.

13. Artegoitia VM, Foote AP, Lewis RM, Freetly HC. Rumen Fluid Metabolomics Analysis Associated with Feed Efficiency on Crossbred Steers. Sci Rep-Uk. 2017;7(1):2864.

14. Sun HZ, Plastow G, Guan LL. Invited review: Advances and challenges in application of feedomics to improve dairy cow production and health. J Dairy Sci. 2019;102(7):5853-70.

15. Sun H, Wang B, Wang J, Liu H, Liu J. Biomarker and pathway analyses of urine metabolomics in dairy cows when corn stover replaces alfalfa hay. J Anim Sci Biotechno. 2016;7(1):49.

16. Dervishi E, Zhang G, Zwierzchowski G, Mandal R, Wishart DS, Ametaj BN. Serum metabolic fingerprinting of pre-lameness dairy cows by GC-MS reveals typical profiles that can identify susceptible cows. J Proteomics. 2020;213:103620.

17. Van Soest PJ, Robertson JB, Lewis BA. Methods for dietary fiber, neutral detergent fiber, and nonstarch polysaccharides in relation to animal nutrition. J Dairy Sci. 1991;74(10):3583-97.

18. AOAC. Official Methods of Analysis of AOAC International. 14th ed. Vol. 1. Arlington: Association of Official Analytical Chemists; 1990.

19. FAN C, SU D, TIAN H, HU R, YANG RANL. Y et al. Milk production and composition and metabolic alterations in the mammary gland of heat-stressed lactating dairy cows. J Integr Agr. 2019;18(12):2844-53.

20. Ammer S, Lambertz C, von Soosten D, Zimmer K, Meyer U, Dänicke S, et al. Impact of diet composition and temperature-humidity index on water and dry matter intake of high-yielding dairy cows. J Anim Physiol An N. 2018;102(1):103-13.

21. Wheelock JB, Rhoads RP, Vanbaale MJ, Sanders SR, Baumgard LH. Effects of heat stress on energetic metabolism in lactating Holstein cows. J Dairy Sci. 2010;93(2):644-55.

22. Chacher B, Zhu W, Ye JA, Wang DM, Liu JX. Effect of dietary N-carbamoylglutamate on milk production and nitrogen utilization in high-yielding dairy cows. J Dairy Sci. 2014;97(4):2338-45.

23. Gu FF, Liang SL, Wei ZH, Wang CP, Liu HY, Liu JX, et al. Short communication: Effects of dietary addition of Ncarbamoylglutamate on milk composition in mid-lactating dairy cows. J Dairy Sci. 2018;101(12):10985-90.

24. Wheelock JB, Rhoads RP, Vanbaale MJ, Sanders SR, Baumgard LH. Effects of heat stress on energetic metabolism in lactating Holstein cows. J Dairy Sci. 2010;93(2):644-55.

25. Sun Y, Liu J, Ye G, Gan F, Hamid M, Liao S, et al. Protective effects of zymosan on heat stress-induced immunosuppression and apoptosis in dairy cows and peripheral blood mononuclear cells. Cell Stress Chaperon. 2018;23(5):1069-78.

26. DahI GE, Tao S, Monteiro APA. Effects of late-gestation heat stress on immunity and performance of calves. J Dairy Sci. 2016;99(4):3193-8.

27. Li S, Tan H, Wang N, Zhang Z, Lao L, Wong C, et al. The Role of Oxidative Stress and Antioxidants in Liver Diseases. Int J Mol Sci. 2015;16(11):26087-124.

28. Xiao L, Cao W, Liu G, Fang T, Wu X, Jia G, et al. Arginine, N-carbamylglutamate, and glutamine exert protective effects against oxidative stress in rat intestine. Anim Nutr. 2016;2(3):242-8.

Page $11 / 14$ 
29. Mo W, Wu X, Jia G, Zhao H, Chen X, Tang J, et al. Roles of dietary supplementation with arginine or N-carbamylglutamate in modulating the inflammation, antioxidant property, and mRNA expression of antioxidant-relative signaling molecules in the spleen of rats under oxidative stress. Anim Nutr. 2018;4(3):322-8.

30. Zhang H, Zhao F, Peng A, Dong L, Wang M, Yu L, et al. Effects of Dietary I-Arginine and N-Carbamylglutamate Supplementation on Intestinal Integrity, Immune Function, and Oxidative Status in Intrauterine-Growth-Retarded Suckling Lambs. J Agr Food Chem. 2018;66(16):4145-54.

31. Hu JY, Hester PY, Makagon MM, Xiong Y, Gates RS, Cheng HW. Effect of cooled perches on physiological parameters of caged White Leghorn hens exposed to cyclic heat. Poultry Sci. 2019;98(6):2317-25.

32. Leyva-Corona JC, Reyna-Granados JR, Zamorano-Algandar R, Sanchez-Castro MA, Thomas MG, Enns RM, et al. Polymorphisms within the prolactin and growth hormone/insulin-like growth factor- 1 functional pathways associated with fertility traits in Holstein cows raised in a hot-humid climate. Trop Anim Health Pro. 2018;50(8):1913-20.

33. Allen JD, Hall LW, Collier RJ, Smith JF. Effect of core body temperature, time of day, and climate conditions on behavioral patterns of lactating dairy cows experiencing mild to moderate heat stress. J Dairy Sci. 2015;98(1):118-27.

34. Tian H, Wang W, Zheng N, Cheng J, Li S, Zhang Y, et al. Identification of diagnostic biomarkers and metabolic pathway shifts of heat-stressed lactating dairy cows. J Proteomics. 2015;125:17-28.

35. Liu G, Xiao L, Cao W, Fang T, Jia G, Chen X, et al. Changes in the metabolome of rats after exposure to arginine and Ncarbamylglutamate in combination with diquat, a compound that causes oxidative stress, assessed by 1H NMR spectroscopy. Food Funct. 2016;7(2):964-74.

36. Li D, Wang X, Liu B, Liu Y, Zeng Z, Lu L, et al. Exercises in hot and humid environment caused liver injury in a rat model. Plos One. 2014;9(12):e111741.

37. Song X, Wang J, Wang P, Tian N, Yang M, Kong L. (1)H NMR-based metabolomics approach to evaluate the effect of Xue-FuZhu-Yu decoction on hyperlipidemia rats induced by high-fat diet. J Pharm Biomed Anal. 2013;78-79(202 - 10.

38. Panunzi S, De Gaetano A, Mingrone G. Approximate linear confidence and curvature of a kinetic model of dodecanedioic acid in humans. Am J Physiol Endoc M. 2005;289(5):E915-22.

39. Hara E. Relationship between Obesity, Gut Microbiome and Hepatocellular Carcinoma Development. Dig Dis. 2015;33(3):34650.

40. Natella F, Nardini M, Di Felice M, Scaccini C. Benzoic and cinnamic acid derivatives as antioxidants: structure-activity relation. J Agr Food Chem. 1999;47(4):1453-9.

\section{Figures}




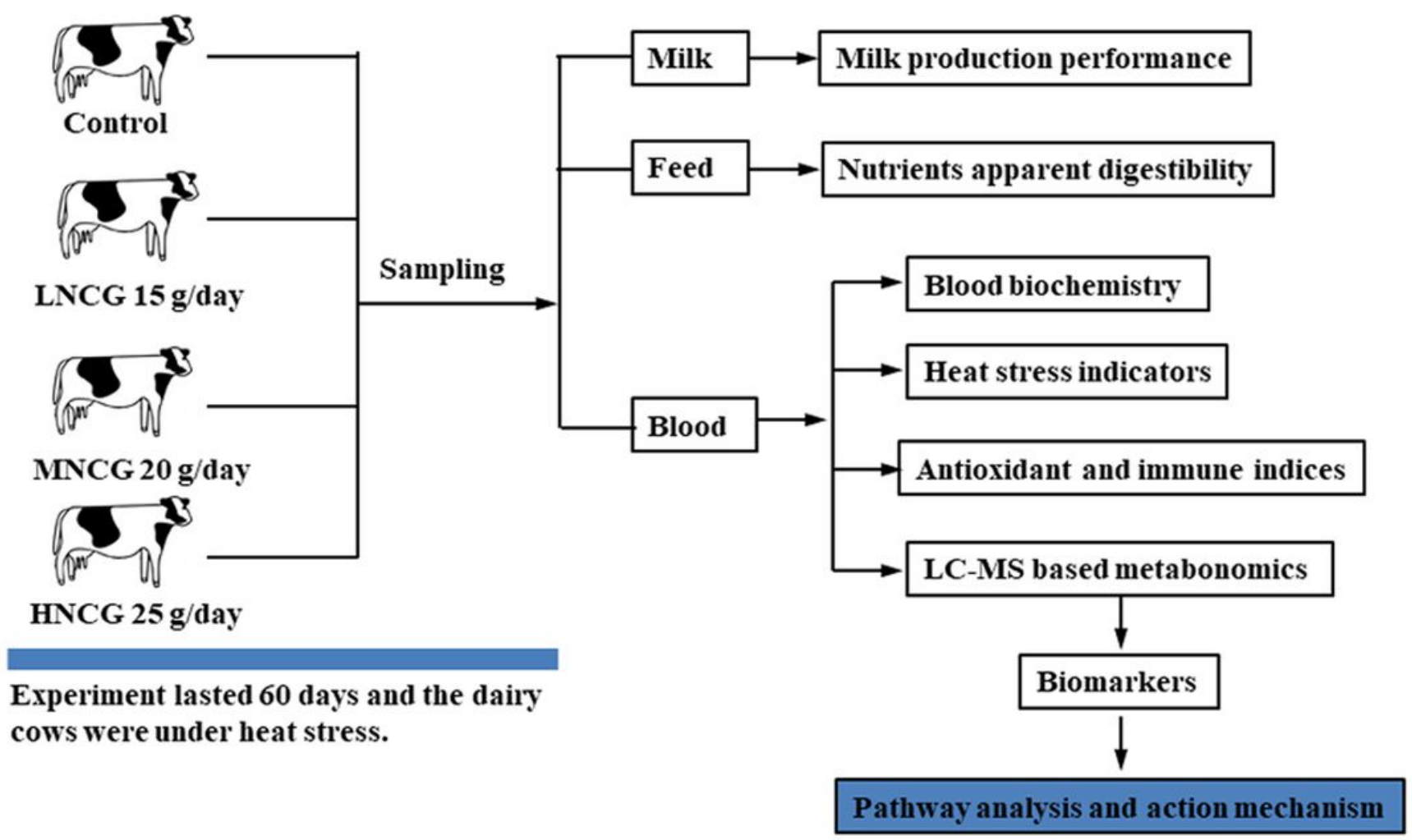

Figure 1

Flowchart of the experiment procedure.

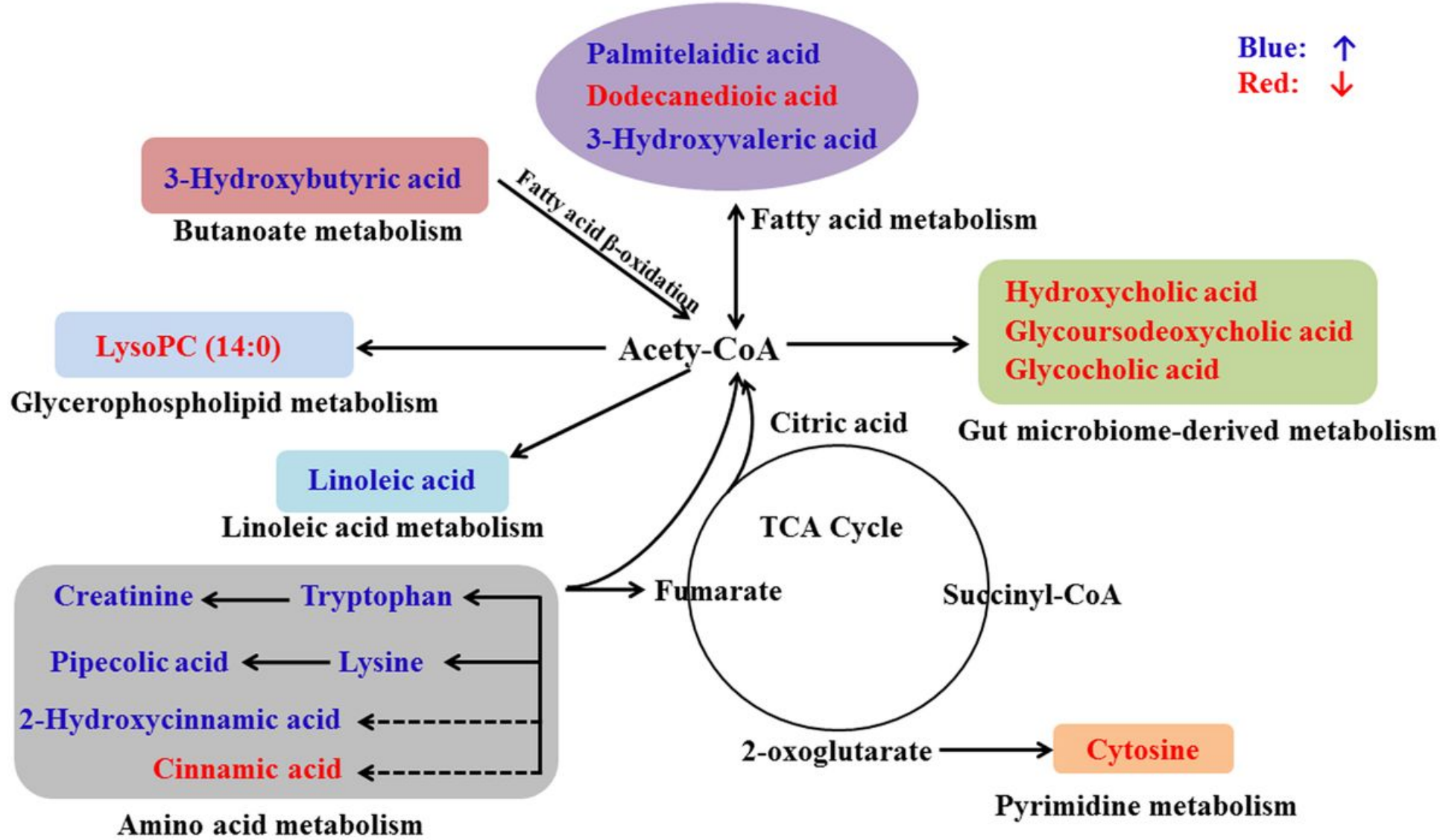


Figure 2

Clustering heatmap of the different metabolites and the metabolomics pathway analysis. A: Pearson's correlations and clustering heatmap of the different plasma metabolites. Metabolites were represented in rows and the samples from different group were represented in columns. Increasing intensities of the metabolites were indicated with blue to red colors. B: Pathway analysis performed by MetaboAnalyst Software based on the KEGG pathway library of Bos Taurus.
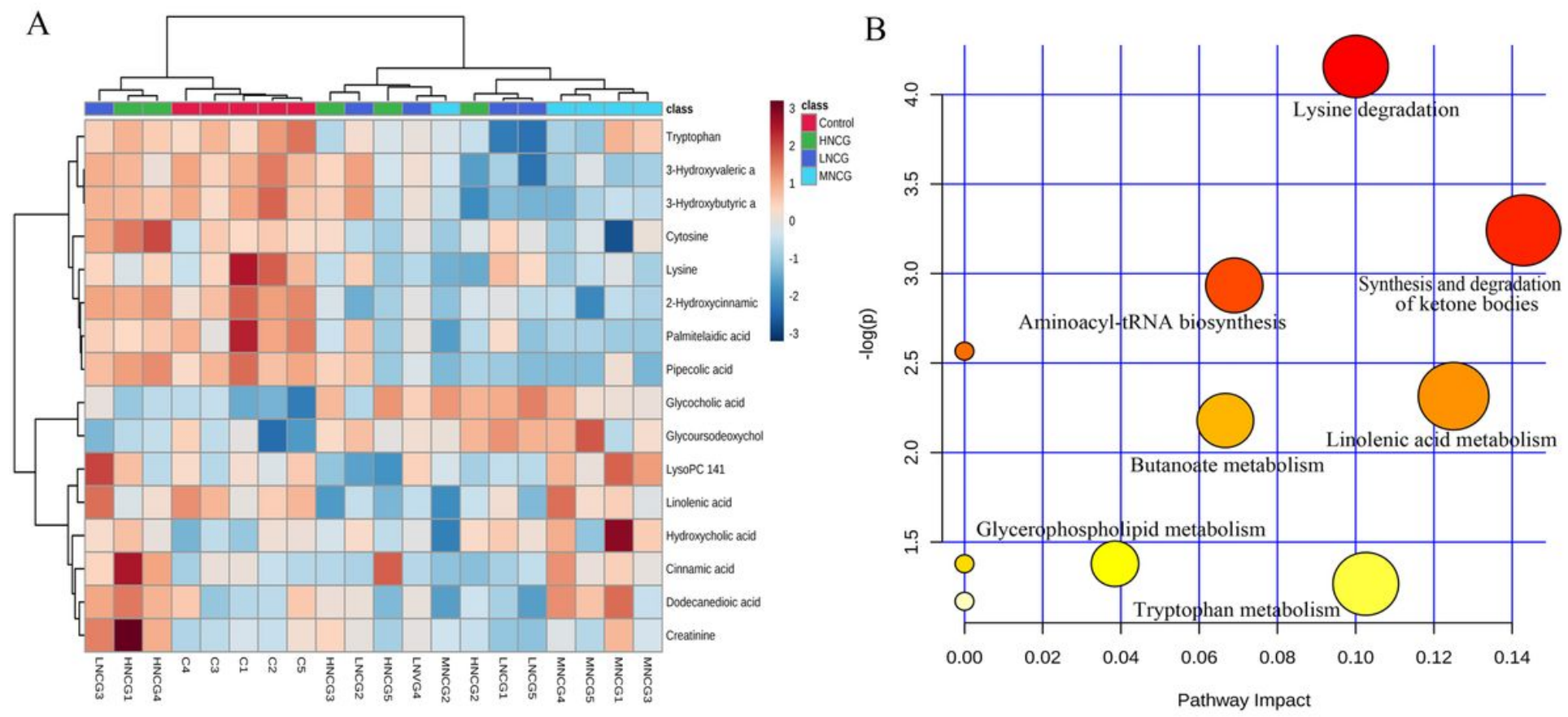

Figure 3

Integrative plasma metabolic pathway changes on the dairy cows under HS with NCG treatment. Potential metabolic pathways related with NCG in dairy cows under HS were established. Compared with the control group, change trends of the metabolites in NCG treatment groups were presented by different color that the blue and red indicated the increase and reduction, respectively.

\section{Supplementary Files}

This is a list of supplementary files associated with this preprint. Click to download.

- SupplementFiles.docx

- SupplementFiles.docx

- SupplementFiles.docx 